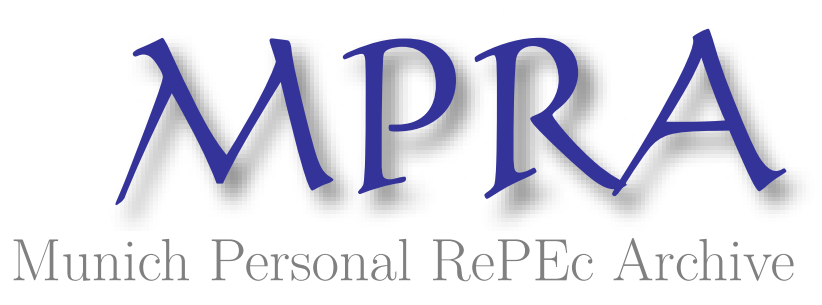

\title{
The Effect of Spillovers on the Provision of Local Public Goods
}

Bloch, Francis and Zenginobuz, Unal

15 December 2004

Online at https://mpra.ub.uni-muenchen.de/186/

MPRA Paper No. 186, posted 07 Oct 2006 UTC 


\title{
Forthcoming in Review of Economic Design
}

\section{The Effect of Spillovers on the Provision of Local Public Goods}

\author{
Francis Bloch* and Unal Zenginobuz ${ }^{\dagger}$
}

October 5, 2006

\begin{abstract}
This paper analyzes the provision of local public goods with positive spillovers across jurisdictions. If spillovers are symmetric, the noncooperative game played by jurisdictions admits a unique equilibrium, and an increase in spillovers reduces the total provision of public goods. Smaller jurisdictions always reduce their contribution, but larger jurisdictions can increase their contribution. When spillovers are asymmetric, equilibrium is unique if spillovers are low, while multiple equilibria exist for high spillover values. In the case of two jurisdictions, an increase in the flow of spillovers to one jurisdiction benefits agents from that jurisdiction but harms agents in the other jurisdiction. Beyond the case of two jurisdictions, the effect of changes in spillovers cannot be signed. An increase in the spillovers flowing to a jurisdiction can actually result in an increase in the supply of public goods by that jurisdiction and harm agents residing in it, while benefiting agents in the other jurisdictions. The results of the paper reveal the complexity of interactions that will plague the design of institutions for multijurisdictional local public good economies with spillovers.
\end{abstract}

JEL CLASSIFICATION NUMBERS: H41, H73, H77

KEYwords:Local Public Goods, Positive Spillovers, Equilibrium

\footnotetext{
*GREQAM and Université de la Méditerranée, 2 rue de la Charité, 13236 Marseille CEDEX 02, France. bloch@ehess.univ-mrs.fr

†Department of Economics and Center for Economic Design, Bogazici University, 34342 Bebek, Istanbul, Turkey. zenginob@boun.edu.tr
} 


\section{Introduction}

The provision of public goods is one of the prime applications of economic design. Since the 1960's, considerable efforts have been expanded in designing rules and institutions in order to elicit information about agents' preferences for public goods, or to encourage private contributions to public goods. For the most part, the theoretical and empirical literature in economics has focused on two polar models: the provision of pure public goods which benefit all agents, and the provision of local public goods which only benefit agents in one community.

In this paper, we focus on the "intermediate" case, where agents are partitioned into communities, but enjoy positive spillovers from the public goods provided in different communities. Of course, the presence of spillovers across jurisdictions has long been noted by economists. For example, suburban residents benefit from amenities provided in central cities; communities often build joint infrastructures which benefit residents in all communities; in the case of transfrontier pollution, countries benefit from pollution abatement programs of other countries. In the late 1960's, a series of theoretical studies on spillovers ((Williams, 66), Brainard and Dolbaer (1967), Boskin (1973)) culminated in Oates (1972)'s study of fiscal federalism, which points out conditions under which central provision of local public goods with spillovers should be preferred to a decentralized system. ${ }^{1}$

The objective of this paper is to revisit the issue of local public provision with spillovers using modern game-theoretic tools. Assuming that local governments independently choose the level of public goods (or alternatively the proportional tax rate), the provision of public goods results from the non-cooperative equilibrium of a game played by jurisdictions. We analyze the properties of this equilibrium, focussing on uniqueness and the comparative statics effects of changes in the structure of spillovers. ${ }^{2}$

\footnotetext{
${ }^{1}$ Following Oates (1972)'s seminal contribution, the literature on fiscal federalism has expanded rapidly. For recent contributions emphasizing inter-jurisdictional spillovers, see Lockwood (2002), Besley and Coate (2003), Cremer and Palfrey (2003) and Redoano and Scharf (2004).

${ }^{2}$ We emphasize that our analysis is primarily positive: we characterize equilibrium for a
} 
In order to fix ideas, consider the following problem. Residents in one community can benefit from amenities (restaurants, opera houses, museums, etc..) offered in different communities. The intensity of spillovers reflects the transportation costs to move across cities. The questions we raise are the following: When is the amount of local public goods provided by different communities unique? Does a reduction in the transportation cost (either a uniform reduction, or a reduction for a specific city) affect the amount of public goods and the utility of residents in different cities?

We first consider the case of symmetric spillovers, which are parametrized by a single parameter $\alpha \in[0,1]$ - with the understanding that residents in one community only benefit from a fraction $\alpha$ of the public goods provided in other communities. In this symmetric model, we show (adapting the well-known argument of Bergstrom, Blume and Varian (1986) and (1992)), that equilibrium is always unique. Furthermore, an increase in the spillover parameter always reduces the total provision of public goods. However, interestingly, an increase in $\alpha$ does not necessarily reduce the provision of public goods in all communities. We show that smaller communities will always reduce their provision of public good (and also see their utility increase), but that larger jurisdiction can in fact increase their supply of public goods, and be harmed by an increase in the spillover parameter.

In the second part of the paper, we turn our attention to the general case, where spillovers between jurisdictions are represented by a general matrix $A=$ $\left[\alpha_{i j}\right]$. We first show that when spillovers are low (i.e. $\sum_{j \neq i} \alpha_{i j}<1$ for all $i$ ), equilibrium is unique. For higher spillovers, the game of public good provision can in fact exhibit multiple equilibria. In the general case, the level of public good is determined by a complex interplay between the sizes of jurisdictions and the spillover matrix, and the effect of changes in spillovers are difficult to ascertain. In the specific case of two communities, we show that an increase in

fixed distribution of the population across jurisdictions and a general spillover structure. We do not attempt to study normative implications of the model, and do not consider public policies (such as inter-jurisdictional transfers or centralization) which could alleviate the inefficiencies due to externalities. 
the flow of spillovers to one jurisdiction reduces the level of public good provision of that jurisdiction, and raises the level of public good provision in the other. This results in a higher utility for agents which have experienced an increase in the spillover, but a lower utility for agents in the other jurisdiction. Beyond the case of two jurisdictions, the effect of changes in spillovers cannot be signed. We provide an example to show that an increase in the spillovers flowing to a jurisdiction can actually result in an increase in the supply of public goods of that jurisdiction and harm agents residing in it (while benefiting agents in the other jurisdictions).

Returning to our illustration, the results of the paper can thus be interpreted as follows. Suppose that two cities (like Paris and Lyon in France, or Ankara and Istanbul in Turkey) are linked by a new high speed train line. This amounts to a global reduction in transportation costs between the two cities, and an increase in the intensity of spillovers. Our results suggest that as a consequence, the provision of public goods in the smaller jurisdictions (Ankara and Lyon) will decrease, whereas the amount of public goods provided in the larger jurisdictions (Paris and Istanbul) might go up. Residents in Ankara and Lyon will definitely benefit from the new high speed train line, but residents in Paris and Istanbul may be hurt.

Before we turn to the description of the model, we should note that our specification (or variants of it) have already been used in different contexts. Lockwood (2002) considers a model with the same specification of spillovers, but with discrete public projects, and analyzes fiscal federalism. Bjortvatn and Schjelderup (2002) use the model with symmetric spillovers to analyze competition in capital taxes. In a companion paper, (Bloch and Zenginobuz (2004)), we use the same model to analyze mobility across jurisdictions and characterize Tiebout equilibria as a function of the spillovers across jurisdictions.

The rest of the paper is organized as follows. The next Section presents the model and notations. Section 3 analyzes the game with symmetric spillovers. Section 4 considers asymmetric spillovers, and Section 5 contains our conclusions. 


\section{The Model}

Agents and Jurisdictions

We consider an economy with a continuum of identical agents on the interval $[0,1]$ with Lebesgue measure $\lambda$. There are $n$ distinct fixed jurisdictions in the economy, indexed by $i=1,2, \ldots, n$. An assignment of agents to jurisdictions is a measurable mapping $\mathcal{A}:[0,1] \rightarrow\{1,2, \ldots, n\}$. We define the distribution of the population across jurisdictions by a vector $\mathbf{m}$ in the $n-1$-dimensional simplex, $\mathbf{m} \in \Delta=\left\{\left(m_{1}, m_{2}, \ldots, m_{n}\right), m_{i} \geq 0, \sum m_{i}=1\right\}$ where $m_{i}=\lambda(\{a, \mathcal{A}(a)=i\})$ denotes the fraction of the population living in jurisdiction $i$.

There are two goods in the economy: a private consumption good $c$ and a local public good provided within each jurisdiction. Each jurisdiction $i$ chooses the amount of total public goods, $G_{i}$ in order to maximize the sum of utilities of agents in that jurisdictions. (Because agents are identical, this is equivalent to choosing a uniform tax rate $t_{i}$ on the consumption good). We depart from traditional models of local public goods by assuming that there exist positive spillovers across jurisdictions. Specifically, we suppose that spillovers are parametrized by a matrix $A=\left[\alpha_{i j}\right]$ where $0 \leq \alpha_{i j} \leq 1$ denotes the spillovers from jurisdiction $i$ to jurisdiction $j$. We interpret $\alpha_{i j}$ as the degree of spillovers that enable an agent in jurisdiction $i$ to consume the local public good provided in jurisdiction $j$. All spillovers are assumed to be positive and $\alpha_{i i}=1$ for all $i$. The two special cases where $\alpha_{i j}=0$ for all $i \neq j$ and $\alpha_{i j}=1$ for all $i \neq j$ correspond to the two polar cases of pure local public goods and pure public goods respectively.

Local public goods provided in different jurisdictions are taken to be perfect substitutes, and we define the composite public good consumed by an agent in jurisdiction $i$ as

$$
\gamma_{i}=\sum_{j} \alpha_{i j} G_{j}
$$

All agents share the same utility function over the private good and the composite public good given by

$$
u(c, \gamma)
$$


where $u$ is is twice continuously differentiable, strictly increasing in both arguments. We define the marginal rate of substitution of the consumption good for the public good by

$$
f(c, \gamma)=\frac{u_{c}(c, \gamma)}{u_{\gamma}(c, \gamma)} .
$$

All agents are endowed with one unit of the private good, which can be transformed into public goods through a linear production function. Letting $g$ denote the contribution of an agent to the local public good, we have $c=1-g$. We assume the following property of the utility function.

Assumption 1 The utility function is concave in the private good. Both goods are weakly normal, and one of the two goods is strongly normal.

Assumption 2 The function u satisfies the following boundary conditions: (i) $\lim _{\gamma \rightarrow 0} f(c, \gamma)=0$ for all $c$ and (ii) $\lim _{c \rightarrow 0} f(c, m) \geq m$ for all $m \in[0,1]$.

Assumption 1 guarantees that the marginal rate of substitution $f$ is nonincreasing in $c$ and nondecreasing in $\gamma$. Strong normality of one of the two goods guarantees that $f$ is either decreasing in $c$ (if the public good is strongly normal), or increasing in $\gamma$ (if the consumption good is strongly normal). The boundary conditions of assumption 2 guarantee that, in the case of pure local public goods, any jurisdiction of size $m$ chooses an interior level of public good provision, $G \in(0, m)$. As all agents are identical, and the utility function is concave in the private good, efficient provision of local public goods requires that all agents inside the same jurisdiction $i$ provide the same amount $g_{i}$ and

$$
G_{i}=\int_{a, \mathcal{A}(a)=i} g_{i} d \lambda=m_{i} g_{i}
$$

Hence the utility of an agent in jurisdiction $i$ can be rewritten (in an indirect form) as:

$$
u\left(1-\frac{G_{i}}{m_{i}}, \sum_{j} \alpha_{i j} G_{j}\right) .
$$

Equilibrium 
We suppose that jurisdiction sizes are fixed, and consider the noncooperative game played across jurisdictions. We first define a Nash equilibrium of the noncooperative game.

Definition 1 An equilibrium is a vector $\mathbf{G}$ in $\Re_{+}^{n}$ satisfying: $G_{i} \in \arg \max u(1-$ $\left.\frac{G_{i}}{m_{i}}, \sum_{j} \alpha_{i j} G_{j}\right)$ for all $i$.

Our first result shows that, under Assumption A1, the noncooperative game always admits a pure strategy Nash equilibrium, and provides a characterization of equilibrium in terms of first order conditions.

Proposition 1 Let the utility function satisfy Assumptions A1 and A2. Then the game of public good provision admits a pure strategy Nash equilibrium. Furthermore, jurisdiction i's equilibrium public good provision is characterized by the first order condition:

$$
f\left(1-\frac{G_{i}}{m_{i}}, \gamma_{i}\right)=m_{i}
$$

or

$$
G_{i}=0 \text { and } f\left(1, \gamma_{i}\right) \geq m_{i}
$$

Proof. We first note that, given that jurisdiction $i$ can at most invest its total endowment, $m_{i}$, in the public good, the strategy spaces are compact intervals, $S_{i}=\left[0, m_{i}\right]$. Furthermore, we can characterize the best response function of jurisidiction $i$ by considering the derivative of the indirect utility with respect to $G_{i}$,

$$
\frac{d u}{d G_{i}}=-\frac{1}{m_{i}} u_{c}\left(1-\frac{G_{i}}{m_{i}}, \sum_{j} \alpha_{i j} G_{j}\right)+u_{\gamma}\left(1-\frac{G_{i}}{m_{i}}, \sum_{j} \alpha_{i j} G_{j}\right) .
$$

Normality of the two goods (Assumption A1) guarantees that, at the optimum,

$$
\begin{aligned}
\frac{d^{2} u}{d G_{i}^{2}} & =\left(\frac{1}{m_{i}}\right)^{2} u_{c c}-\frac{2}{m_{i}} u_{c \gamma}+u_{\gamma \gamma} \\
& =\left(\frac{1}{u_{c}}\right)^{2}\left(u_{\gamma}^{2} u_{c c}-2 u_{c} u_{\gamma} u_{c \gamma}+u_{c}^{2} u_{\gamma \gamma}\right)<0 .
\end{aligned}
$$

Hence, the indirect utility is strictly quasi-concave in $G_{i}$ and continuous in $G_{j}$, guaranteeing the existence of an equilibrium in pure strategies. Furthermore, this equilibrium is characterized by the best response functions of the 
jurisdictions which are equivalent to the (necessary and sufficient) first-order conditions.

Quasi-Linear Utility Functions

Equilibrium can easily be computed for the class of quasi-linear utility functions where

$$
u(c, \gamma)=c+v(\gamma)
$$

with $v^{\prime}(\gamma)>0$ and $v^{\prime \prime}(\gamma)<0$. The boundary conditions are then given by : (i) $\lim _{\gamma \rightarrow 0} v^{\prime}(\gamma)=\infty$ and (ii) $1 \geq m v^{\prime}(m)$ for all $m \in(0,1)$.

Let $\mu_{i}$ denote the level of public good provision of jurisdiction $i$ in the absence of spillovers,

$$
\mu_{i}=\left(v^{\prime}\right)^{-1}\left(\frac{1}{m_{i}}\right)
$$

As $v^{\prime \prime}($.$) is strictly decreasing, \mu_{i}$ is well defined and the boundary conditions guarantee that $\mu_{i}$ belongs to the interval $\left[0, m_{i}\right]$. The best-response function of jurisdiction $i$ can then be computed as

$$
G_{i}=\max \left\{\mu_{i}-\sum_{j \neq i} \alpha_{i j} G_{j}, 0\right\}
$$

and the equilibrium level of public good provision is obtained at a fixed point of the best-response mapping assigning a best response to every jurisdiction.

\section{Symmetric spillovers}

In this Section, we suppose that for all $i \neq j, \alpha_{i j}=\alpha_{j i}=\alpha$. Spillovers are symmetric and parametrized by a single value $\alpha \in[0,1]$. Our first result shows that, in that case, the equilibrium level of public good provision is unique.

Proposition 2 Let the utility function satisfy Assumption A1 (with the public good being strongly normal if $\alpha=1$.) Then the equilibrium level of public good provision, $\mathbf{G}$ is unique.

Proof. Suppose that there exist two distinct equilibria $\mathbf{G}$ and $\mathbf{G}^{\prime}$ and without loss of generality suppose that $G_{1}>G_{1}^{\prime}$. We first show that this implies $\gamma_{1} \leq \gamma_{1}^{\prime}$. 
In fact, suppose by contradiction that $\gamma_{1}>\gamma_{1}^{\prime}$. By Assumption A1,

$$
f\left(1-\frac{G_{1}}{m_{1}}, \gamma_{1}\right)>f\left(1-\frac{G_{1}^{\prime}}{m_{1}}, \gamma_{1}^{\prime}\right),
$$

contradicting the fact that $G_{1}$ and $G_{1}^{\prime}$ satisfy the first order condition, with $G_{1}>G_{1}^{\prime}$. As $\gamma_{1} \leq \gamma_{1}^{\prime}$ and $G_{1}>G_{1}^{\prime}$, there must exist another jurisdiction, say jurisdiction 2 for which $G_{2}^{\prime}>G_{2}$. The same line of argument then shows that $\gamma_{2}^{\prime} \leq \gamma_{2}$. The two inequalities in $\gamma$ can be developed as:

$$
\begin{aligned}
G_{1}+\alpha G_{2}+\alpha \sum_{j>2} G_{j} & \leq G_{1}^{\prime}+\alpha G_{2}^{\prime}+\alpha \sum_{j>2} G_{j}^{\prime} \\
G_{2}^{\prime}+\alpha G_{1}^{\prime}+\alpha \sum_{j>2} G_{j}^{\prime} & \leq G_{2}+\alpha G_{1}+\alpha \sum_{j>2} G_{j} .
\end{aligned}
$$

Adding the two inequalities and rearranging, we obtain:

$$
(1-\alpha)\left(G_{1}-G_{1}^{\prime}\right) \leq(1-\alpha)\left(G_{2}-G_{2}^{\prime}\right)
$$

If $\alpha<1$, this implies that

$$
0<G_{1}-G_{1}^{\prime} \leq G_{2}-G_{2}^{\prime}<0
$$

a contradiction.

If $\alpha=1$, the inequalities imply:

$$
\gamma_{1}=\gamma_{1}^{\prime}
$$

If the public good is strongly normal, $f$ is strictly decreasing in $c$ and hence,

$$
f\left(1-\frac{G_{1}}{m_{1}}, \gamma_{1}\right)>f\left(1-\frac{G_{1}^{\prime}}{m_{1}}, \gamma_{1}^{\prime}\right),
$$

contradicting the fact that $G_{1}$ and $G_{1}^{\prime}$ satisfy the first order condition, with $G_{1}>G_{1}^{\prime}$.

Proposition 2 shows that when spillovers are symmetric, equilibrium is unique. The only exception is the case of pure public goods and quasi-linear utility functions, where the total value of public good provision is uniquely determined, but the distribution across jurisdictions is arbitrary. The proof of Proposition 2 is reminiscent of the proof of uniqueness of equilibrium in the model of pure public 
good provision (Bergstrom, Blume and Varian, 1992). As in the case of private provision of public goods, the proof relies heavily on the normality of the two goods. We also note that the fact that spillovers are symmetric plays a crucial role in the proof of the Proposition.

For a fixed level of spillovers, we can easily show that larger jurisdictions always provide a higher amount of local public goods. Again, this result is not surprising as it parallels well-known arguments in the case of local public goods and pure public goods.

Lemma 1 Consider two jurisdictions with $m_{i}>m_{j}$. Then, in equilibrium, $G_{i} \geq G_{j}$.

Proof. Suppose that $m_{i}>m_{j}$ and $G_{j}>G_{i}$. Using the first order condition,

$$
f\left(1-\frac{G_{i}}{m_{i}}, \gamma_{i}\right) \geq m_{i}>m_{j} \geq f\left(1-\frac{G_{j}}{m_{j}}, \gamma_{j}\right)
$$

However, $1-G_{i} / m_{i}>1-G_{j} / m_{j}$ and $\gamma_{i}=G_{i}+\alpha G_{j}+\alpha \sum_{k \neq i, j} G_{k}<\gamma_{j}=$ $G_{j}+\alpha G_{i}+\alpha \sum_{k \neq i, j} G_{k}$. As $f$ is nonincreasing in $c$, nondecreasing in $\gamma$, and one of the two goods is strongly normal, we have

$$
f\left(1-\frac{G_{i}}{m_{i}}, \gamma_{i}\right)<f\left(1-\frac{G_{j}}{m_{j}}, \gamma_{j}\right),
$$

yielding a contradiction.

\subsection{Changes in the spillover parameter}

We now analyze the effects of changes in the spillover parameter $\alpha$ on the provision of pulbic goods, and derive the main results of the paper. Our first Proposition shows that an increase in the spillover parameter reduces the total provision of public goods, and necessarily increases the utility of agents in some jurisdiction.

Proposition 3 Suppose that the utility function satisfies A1. Then, if $\alpha^{\prime}>\alpha$, the total amount of public goods provided satisfies $\bar{G}=\sum_{i} G_{i} \geq \bar{G}^{\prime}=\sum_{i} G_{i}^{\prime}$. 
Proof. Consider two spillover parameters with $\alpha^{\prime}>\alpha$ and let $\bar{G}$ and $\bar{G}^{\prime}$ denote the corresponding total levels of public good provision. Suppose by contradiction that $\bar{G}^{\prime}>\bar{G}$. Then there exists a jurisdiction $i$ for which $G_{i}^{\prime}>G_{i}$. From the first order conditions:

$$
f\left(1-\frac{G_{i}^{\prime}}{m_{i}},\left(1-\alpha^{\prime}\right) G_{i}^{\prime}+\alpha^{\prime} \bar{G}^{\prime}\right) \leq f\left(1-\frac{G_{i}}{m_{i}},(1-\alpha) G_{i}+\alpha \bar{G}\right) .
$$

But, as $1-\frac{G_{i}^{\prime}}{m_{i}}<1-\frac{G_{i}}{m_{i}}$ and the two goods are normal, this implies that $\left(1-\alpha^{\prime}\right) G_{i}^{\prime}+\alpha^{\prime} \bar{G}^{\prime} \leq(1-\alpha) G_{i}+\alpha \bar{G}$, contradicting the assumptions $G_{i}^{\prime}>G_{i}$ and $\bar{G}^{\prime}>\bar{G}$.

Proposition 3 shows that the total provision of public goods decreases with an increase in the spillover parameter. It does not show however that the provision of public goods decreases for all jurisdictions. The next results will help us identify those jurisdictions for which public good provision decreases.

Proposition 4 Suppose that utilities satisfy Assumption A1 and A2. Then, if $m_{i}<m_{j}$ and $\alpha^{\prime}>\alpha$, if $G_{j}^{\prime} \leq G_{j}$ then $G_{i}^{\prime} \leq G_{i}$.

Proof. Suppose by contradiction that $G_{j}^{\prime} \leq G_{j}$ and $G_{i}^{\prime}>G_{i}$. First consider the case $G_{j}^{\prime}<G_{j}$. Then, by the first order condition:

$$
f\left(1-\frac{G_{j}}{m_{j}}, G_{j}+\alpha \sum_{k \neq j} G_{k}\right) \leq f\left(1-\frac{G_{j}^{\prime}}{m_{j}}, G_{j}^{\prime}+\alpha^{\prime} \sum_{k \neq j} G_{k}^{\prime}\right)
$$

Given that $G_{j}^{\prime}<G_{j}$, and that the two goods are normal, this implies that

$$
G_{j}^{\prime}+\alpha^{\prime} \sum_{k \neq j} G_{k}^{\prime} \geq G_{j}+\alpha \sum_{k \neq j} G_{k} .
$$

Similarly, as $G_{i}^{\prime}>G_{i}$,

$$
G_{i}+\alpha \sum_{k \neq i} G_{k} \geq G_{i}^{\prime}+\alpha^{\prime} \sum_{k \neq i} G_{k}
$$

Summing up these inequalities,

$$
G_{i}+G_{j}^{\prime}+\alpha^{\prime} G_{i}^{\prime}+\alpha G_{j} \geq G_{j}+G_{i}^{\prime}+\alpha^{\prime} G_{j}^{\prime}+\alpha G_{i}
$$


or

$$
\left(1-\alpha^{\prime}\right)\left(G_{j}^{\prime}-G_{i}^{\prime}\right) \geq(1-\alpha)\left(G_{j}-G_{i}\right)
$$

Given that $m_{i}<m_{j}$, by Proposition $1, G_{j}-G_{i} \geq 0$ and $G_{j}^{\prime}-G_{i}^{\prime} \geq 0$. As $\alpha^{\prime}>\alpha$, $\left(1-\alpha^{\prime}\right)<(1-\alpha)$. Hence, the preceding inequality implies:

$$
\left(G_{j}^{\prime}-G_{j}^{\prime}\right) \geq\left(G_{j}-G_{i}\right)
$$

contradicting the assumptions: $G_{j}^{\prime}<G_{j}$ and $G_{i}^{\prime}>G_{i}$.

Suppose now that $G_{j}^{\prime}=G_{j}$. If this is an interior solution, the first order conditions guarantee that:

$$
G_{j}^{\prime}+\alpha^{\prime} \sum_{k \neq j} G_{k}^{\prime}=G_{j}+\alpha \sum_{k \neq j} G_{k}
$$

and the same argument can be applied. If $G_{j}^{\prime}=G_{j}=0$, as $m_{i}<m_{j}, G_{i}=$ $G_{i}^{\prime}=0$ and the result follows. Finally, the boundary condition of Assumption A2 guarantees that $G_{j}=G_{j}^{\prime}=m_{j}$ cannot hold at equilibrium.

Proposition 4 shows that, if the public good provision of some jurisdiction $i$ decreases, then necessarily the public good provision of all smaller jurisdictions must decrease. In particular, as the total public good provision always decreases, the public good provision of the smallest jurisdiction cannot increase. We now turn to equilibrium utilities and show that there exists a direct connection between changes in the provision of public goods and changes in utilities.

Proposition 5 Suppose that the utility function satisfies Assumption A1. Let $\alpha^{\prime} \neq \alpha$. Then, $U_{i}^{\prime}<U_{i}$ if $G_{i}^{\prime}>G_{i}$ and $U_{i}^{\prime}>U_{i}$ if $G_{i}^{\prime}<G_{i}$.

Proof. Suppose that $G_{i}^{\prime}>G_{i}$. From the first order condition, we have:

$$
f\left(1-\frac{G_{i}^{\prime}}{m_{i}^{\prime}}, G_{i}^{\prime}+\alpha^{\prime} \sum_{j \neq i} G_{j}^{\prime}\right) \leq f\left(1-\frac{G_{i}}{m_{i}}, G_{i}+\alpha \sum_{j \neq i} G_{j}\right) .
$$

As $G_{i}^{\prime}>G_{i}$ and the two goods are normal, this implies that:

$$
G_{i}+\alpha \sum_{j \neq i} G_{j} \geq G_{i}^{\prime}+\alpha^{\prime} \sum_{j \neq i} G_{j}^{\prime}
$$


and hence

$$
\alpha \sum_{j \neq i} G_{j}>\alpha^{\prime} \sum_{j \neq i} G_{j}^{\prime}
$$

Now, $u\left(1-\frac{G_{i}}{m_{i}}, G_{i}+\alpha \sum_{j \neq i} G_{j}\right) \geq u\left(1-\frac{G_{i}^{\prime}}{m_{i}}, G_{i}^{\prime}+\alpha \sum_{j \neq i} G_{j}\right)>u\left(1-\frac{G_{i}^{\prime}}{m_{i}}, G_{i}^{\prime}+\right.$ $\left.\alpha^{\prime} \sum_{j \neq i} G_{j}^{\prime}\right)$. The same argument can be applied for $G_{i}^{\prime}<G_{i}$.

Proposition 5 thus show that a decrease in the provision of public goods by some jurisdiction is associated with an increase in the utility of agents belonging to that jurisdiction. By Proposition 3, this shows that an increase in the spillover parameter cannot result in a decrease in utility for all agents. Proposition 4 furthermore shows that agents in the smallest jurisdiction cannot suffer a utility loss if the spillover paramer increases. In fact, if the provision of public goods in the smallest jurisdiction goes down, agents in the smallest jurisdiction always benefit from the increase in the spillover parameter.

We summarize our results as follows. Agents belonging to smaller jurisdiction typically experience a decrease in the provision of public goods and an increase in utility when the spillover paramer increases. Agents in larger jurisdiction may in fact provide more public goods and suffer from a utility loss with an increase in spillovers. We now illustrate these effects by characterizing completely equilibrium in a model with two jurisdictions and quasi-linear utility.

\section{Two Jurisdictions and Quasilinear Utilities}

We consider two jurisdictions, with $m_{1} \leq m_{2}$. In an interior equilibrium,

$$
G_{1}=\frac{\left(\mu_{1}-\alpha \mu_{2}\right)}{1-\alpha^{2}}, G_{2}=\frac{\left(\mu_{2}-\alpha \mu_{1}\right)}{1-\alpha^{2}}
$$

This equilibrium exists as long as $\alpha<\frac{\mu_{1}}{\mu_{2}}<1$. In that interior equilibrium, we easily compute:

$$
\frac{\partial G_{i}}{\partial \alpha}=\frac{2 \alpha \mu_{i}-\left(1+\alpha^{2}\right) \mu_{j}}{\left(1-\alpha^{2}\right)^{2}}, i \neq j,
$$

As $2 \alpha \mu_{1}-\left(1+\alpha^{2}\right) \mu_{2}<0$, the provision of public good of jurisdiction 1 is always decreasing in $\alpha$, and the equilibrium utility increasing. For agents in the larger jurisdiction, the provision of public good is decreasing if and only if 
$\frac{\mu_{1}}{\mu_{2}} \leq \frac{2 \alpha}{1+\alpha^{2}} \leq 1$, and increasing otherwise. A simple computation shows that $\frac{\mu_{1}}{\mu_{2}} \leq \frac{2 \alpha}{1+\alpha^{2}}$ if and only if $\alpha \leq \frac{\mu_{2}}{\mu_{1}}\left[1-\sqrt{1-\left(\frac{\mu_{1}}{\mu_{2}}\right)^{2}}\right]$.

If $\alpha>\frac{\mu_{1}}{\mu_{2}}$, in equilibrium, jurisdiction 2 is the only jurisdiction providing the public good, so $G_{2}=\mu_{2}$ and $G_{1}=0$ for all $\alpha$. In that case, the equilibrium utility of agents in jurisdiction 2 is constant, and the equilibrium utility of agents in jurisdiction 1 is increasing in $\alpha$. We summarize these findings in the following Proposition.

Proposition 6 Suppose that utilities are quasi-linear and satisfy Assumptions $A 1$ and $A 2$, and let $n=2$ with $m_{1} \leq m_{2}$. If $\alpha \leq \frac{\mu_{2}}{\mu_{1}}\left[1-\sqrt{1-\left(\frac{\mu_{1}}{\mu_{2}}\right)^{2}}\right]$, then $G_{1}$ and $G_{2}$ are both decreasing in $\alpha$, and $U_{1}$ and $U_{2}$ are increasing in $\alpha$. If $\frac{\mu_{2}}{\mu_{1}}\left[1-\sqrt{1-\left(\frac{\mu_{1}}{\mu_{2}}\right)^{2}}\right] \leq \alpha \leq \frac{\mu_{1}}{\mu_{2}}$, then $G_{1}$ is decreasing in $\alpha$ but $G_{2}$ increasing, and $U_{1}$ is increasing in $\alpha$ and $U_{2}$ decreasing. If $\alpha \geq \frac{\mu_{1}}{\mu_{2}}$, jurisdiction 2 is the only jurisdiction providing the public good, $G_{1}$ and $G_{2}$ are independent of $\alpha$, and $U_{1}$ is increasing in $\alpha$ and $U_{2}$ independent of $\alpha$.

The following example illustrates Proposition 6 .

Example 1 Let $n=2, m_{1}=1 / 3, m_{2}=2 / 3$ and $u(c, \gamma)=c+\log \gamma$.

One can easily compute: $\frac{\mu_{1}}{\mu_{2}}=1 / 2$ and $\frac{\mu_{2}}{\mu_{1}}\left[1-\sqrt{1-\left(\frac{\mu_{1}}{\mu_{2}}\right)^{2}}\right]=2-\sqrt{3}$. The following graph shows the provision of public good of jurisdictions 1 and 2 for $\alpha \in[0,1 / 2]$.

The solid line represents the public good contribution of jurisdiction 2 (the larger jurisdiction) and the dashed line the public good provision of jurisdiction 1 (the smaller jurisdiction). For jurisdiction 1, the provision of public good is strictly decreasing in $\alpha$ and utility strictly increasing in $\alpha$.For jurisdiction 2 , the provision of public good is first decreasing for $\alpha \in[0,2-\sqrt{3}]$, then increasing for $\alpha \in[2-\sqrt{3}, 1 / 2]$. The utility of agents in jurisdiction 2 is thus first increasing in $\alpha$, then decreasing in $\alpha$. 


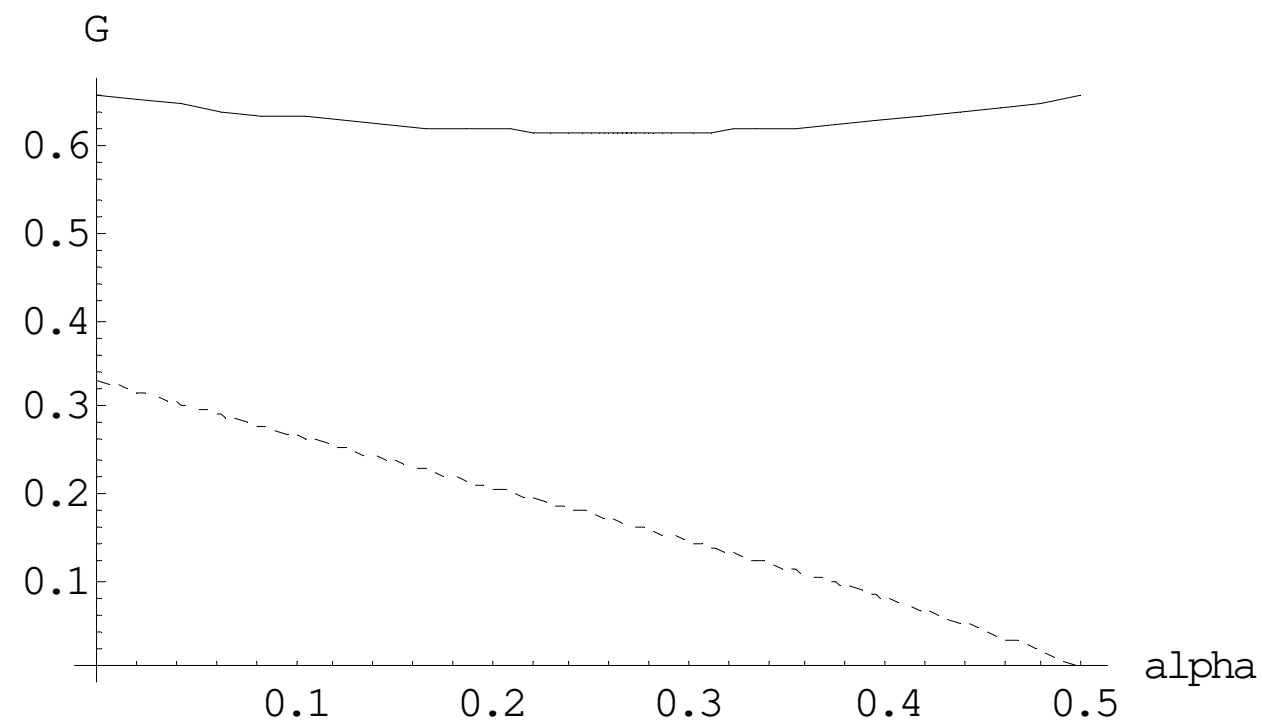

Figure 1: Public good provision in two jurisdictions

\section{Asymmetric Spillovers}

In this Section, we consider the general case where spillovers are given by an arbitrary matrix $A$. We first distinguish between two types of asymmetries. First, for a given pair of jurisdictions, the flows of spillovers may be asymmetric, $\alpha_{i j} \neq \alpha_{j i}$. Second, even if spillovers are symmetric for any pair of jurisdictions, spillovers may be different across different pairs, $\alpha_{i j} \neq \alpha_{k l}$ for $k, l \neq i, j$. It is useful to define a partial order on jurisdictions, in order to determine which jurisdictions benefit more from spillovers.

Definition 2 A jurisdiction $i$ is more central than a jurisdiction $j$ if and only if $\alpha_{i k} \geq \alpha_{j k}$ for all $k \neq i, j$ and $\alpha_{i k}>\alpha_{j k}$ for some $k \neq i, j$.

Our first task is to analyze conditions under which the equilibrium level of public good provision is unique. The following Proposition provides a sufficient condition for uniqueness of equilibrium. 
Proposition 7 Suppose that utility functions satisfy Assumption A1 and that $\sum_{j \neq i} \alpha_{i j}<1$ for all $i$. Then the equilibrium level of public good provision, $\mathbf{G}$, is unique.

Proof. We show that when $\sum_{j \neq i} \alpha_{i j}<1$ for all $i$, the best-response mapping is a contraction. To this end, recall that the best response of a jurisdiction $i$ is given by:

$$
\phi_{i}\left(G_{-i}\right)=\arg \max _{G_{i} \in\left[0, m_{i}\right]} u_{i}\left(1-\frac{G_{i}}{m_{i}}, G_{i}+\sum \alpha_{i j} G_{j}\right) .
$$

Using the first-order condition

$$
\begin{array}{cccccc} 
& \phi_{i}\left(G_{-i}\right)=0 & \text { if } & f\left(1, \sum \alpha_{i j} G_{j}\right) & \geq 0, \\
\text { or } & \phi_{i}\left(G_{-i}\right)=m_{i} & \text { if } & f\left(0, m_{i}+\sum \alpha_{i j} G_{j}\right) & \leq m_{i} \\
\text { or } & \phi_{i}\left(G_{-i}\right) & \text { s.t. } & f\left(1-\frac{G_{i}}{m_{i}}, \sum \alpha_{i j} G_{j}\right) & = & m_{i}
\end{array}
$$

The best response mapping $\phi_{i}$ is differentiable everywhere except at the two points where $f\left(1, \sum \alpha_{i j} G_{j}\right)=0$ and $f\left(0, m_{i}+\sum \alpha_{i j} G_{j}\right)=m_{i}$, where the left hand side and right hand side derivatives may be different. This will not affect the argument, and at these two points, we define the derivative to be the right hand side and left hand side derivatives, respectively. If the best response is a corner point $\left(\phi_{i}\left(G_{-i}\right)=0\right.$ or $\left.\phi_{i}\left(G_{-i}\right)=m_{i}\right), \partial \phi_{i} / \partial G_{j}=0$ for all $j$ so that

$$
\sum_{j \neq i} \frac{\partial \phi_{i}}{\partial G_{j}}=0<1
$$

At interior best responses, we compute:

$$
\frac{\partial \phi_{i}}{\partial G_{j}}=\frac{\alpha_{i j} f_{\gamma}}{-f_{c} / m_{i}+f_{\gamma}}
$$

Hence,

$$
\sum_{j \neq i} \frac{\partial \phi_{i}}{\partial G_{j}}=\frac{\sum_{j \neq i} \alpha_{i j} f_{\gamma}}{-f_{c} / m_{i}+f_{\gamma}}>-1
$$

This computation shows that in the Hessian matrix of cross-derivatives, all offdiagonal terms are dominated by the diagonal terms - the dominant diagonal condition holds. This is a sufficient condition for the best-response mapping to be a contraction (see, for example Vives (2000), p. 48), and the equilibrium is unique. 
Proposition 7 shows that when the spillover parameters are sufficiently low, the equilibrium level of public good provision is unique. If there are only two jurisdictions and the good is not a pure public good $\left(\alpha_{i j}, \alpha_{j i}<1\right)$, this result demonstrates that equilibrium is always unique. However, in general, one cannot expect that the economy admits a unique equilibrium level of public good provision. The following example, using three jurisdictions, shows that multiple equilibria can exist.

Example 2 Let $n=3, u(c, \gamma)=c+\log \gamma, m_{1}<m_{2}<m_{3}$ with $m_{3}<m_{1}+m_{2}$. $A=\left[\begin{array}{lll}1 & 0 & \alpha \\ 0 & 1 & \alpha \\ \alpha & \alpha & 1\end{array}\right]$

We show that for $\alpha$ close to 1 , there exist two distinct equilibria, with different levels of public good provision.

Equilibrium 1: All jurisdictions contribute. The contributions are given by the solutions to the system of equations:

$$
\begin{aligned}
G_{1}+\alpha G_{3} & =m_{1}, \\
G_{2}+\alpha G_{3} & =m_{2}, \\
\alpha G_{1}+\alpha G_{2}+G_{3} & =m_{3} .
\end{aligned}
$$

We can easily compute:

$$
\begin{aligned}
G_{1} & =\frac{\alpha m_{3}-\alpha^{2} m_{2}-\left(1-\alpha^{2}\right) m_{1}}{2 \alpha^{2}-1} \\
G_{2} & =\frac{\alpha m_{3}-\alpha^{2} m_{1}-\left(1-\alpha^{2}\right) m_{2}}{2 \alpha^{2}-1} \\
G_{3} & =\frac{\alpha m_{2}+\alpha m_{1}-m_{3}}{2 \alpha^{2}-1}
\end{aligned}
$$

For $\alpha=1$, all contributions are positive, as $G_{1}=m_{3}-m_{2}>0, G_{2}=m_{3}-m_{1}>$ 0 and $G_{3}=m_{1}+m_{2}-m_{3}>0$. As the contributions are continuous in $\alpha$, there exists $\underline{\alpha}<1$ such that all contributions are positive for $\alpha \geq \underline{\alpha}$. Note that in this equilibrium,

$$
\bar{G}=G_{1}+G_{2}+G_{3}=\frac{(2 \alpha-1) m_{3}-(1-\alpha)\left(m_{1}+m_{2}\right)}{2 \alpha^{2}-1}
$$


Equilibrium 2: Jurisdiction 3 is the only one to contribute. In that case,

$$
G_{3}^{\prime}=m_{3}
$$

and as long as $\alpha m_{3}>m_{2}>m_{1}$, neither members of jurisdictions 1 and 2 choose to contribute. We note that these two equilibria are not equivalent in terms of total contribution to the public good. One can easily show that for $\alpha \in\left(\frac{1}{2} \frac{m_{1}+m_{2}}{m_{3}}, 1\right)$

$$
\bar{G}=\frac{(2 \alpha-1)\left(m_{3}+(1-\alpha)\left(m_{1}+m_{2}\right)\right)}{2 \alpha^{2}-1}>m_{3}=\bar{G}^{\prime} .
$$

Example 2 is interesting because it shows that multiple equilibria can exist when the level of spillovers is high enough $\left(\sum \alpha_{i j}>1\right)$, and spillovers are asymmetric $\left(\alpha_{i j} \neq \alpha_{i k}\right)$. In this example, the interior equilibrium (equilibrium 1 ) is unique, and the multiplicity stems from the fact that the game admits both an inteiror and a corner equilibrium.

While the previous example shows that multiple equilibria may exist, we now investigate whether, for a given equilibrium, the level of public good provision can be ranked according to the size of jurisdictions and the structure of spillovers. When spillovers are symmetric, Lemma 1 shows that larger jurisdictions provide more public goods. With asymmetric spillovers, this need not be the case. In fact, Example 2 shows that larger jurisdictions may provide less public goods. If $m_{3}>m_{1}+m_{2} / 2$, then for $\alpha$ close enough to $1, G_{3}<G_{2}$. When spillovers are asymmetric, contributions to the public good depend on the complex interplay between jurisdiction sizes and the spillovers. If jurisdictions have equal size, the following Lemma provides a partial ranking for the contributions to the public good.

Lemma 2 Suppose that $m_{i}=m_{j}$ and jurisdiction $i$ is more central than jurisdiction $j$, then at any equilibrium $G_{i} \leq G_{j}$.

Proof. Let $m_{i}=m_{j}, \alpha_{i k} \geq \alpha_{j k}$ for all $k \neq i, j$ and $\alpha_{i k}>\alpha_{j k}$ for some $k$. Suppose by contradiction that $G_{i}>G_{j}$. Then from the first order condition,

$$
f\left(1-\frac{G_{j}}{m_{j}}, \gamma_{j}\right) \geq m_{j}=m_{i} \geq f\left(1-\frac{G_{i}}{m_{i}}, \gamma_{i}\right) .
$$


However, $1-G_{j} / m_{j}>1-G_{i} / m_{i}$ and $\gamma_{j}=G_{j}+\alpha_{j i} G_{i}+\sum_{k \neq i, j} \alpha_{j k} G_{k}<\gamma_{i}=$ $G_{i}+\alpha_{i j} G_{j}+\sum_{k \neq i, j} \alpha_{i k} G_{k}$. Hence, by normality of the two goods,

$$
f\left(1-\frac{G_{j}}{m_{j}}, \gamma_{j}\right)<f\left(1-\frac{G_{i}}{m_{i}}, \gamma_{i}\right)
$$

contradicting the previous inequality.

Lemma 2 captures a very simple intuition. If two jurisdictions have equal size, but one benefits more from the public goods provided in other jurisdictions, the jurisdiction which is more centrally located contributes less public goods.

\subsection{Changes in the spillover parameter}

We now consider the effect of changes in spillovers in the asymmetric case. We start the analysis with the case of two jurisdictions, with asymmetric spillover flows. We suppose that the change only affects the spillover flow in one direction.

Proposition 8 Let $n=2$. Suppose that the utility function satisfies Assumption $A 1$ and that $\alpha_{i j}^{\prime}>\alpha_{i j}$. Then $G_{i}^{\prime} \leq G_{i}, G_{j}^{\prime} \geq G_{j}, U_{i}^{\prime} \geq U_{i}$ and $U_{j}^{\prime} \leq U_{j}$.

Proof. Suppose by contradiction that $G_{i}^{\prime}>G_{i}$. From the first order condition,

$$
f\left(1-\frac{G_{i}}{m_{i}}, G_{i}+\alpha_{i j} G_{j}\right) \geq f\left(1-\frac{G_{i}^{\prime}}{m_{i}}, G_{i}^{\prime}+\alpha_{i j}^{\prime} G_{j}^{\prime}\right) .
$$

By a familiar argument, this implies that

$$
G_{i}+\alpha_{i j} G_{j} \geq G_{i}^{\prime}+\alpha_{i j}^{\prime} G_{j}^{\prime}
$$

Hence, $G_{j}>G_{j}^{\prime}$ and similarly we obtain:

$$
G_{j}^{\prime}+\alpha_{j i} G_{i}^{\prime} \geq G_{j}+\alpha_{j i} G_{i}
$$

Summing up these two inequalities, we obtain:

$0>\left(G_{i}-G_{i}^{\prime}\right)\left(1-\alpha_{j i}\right) \geq G_{j}\left(1-\alpha_{i j}\right)-G_{j}^{\prime}\left(1-\alpha_{i j}^{\prime}\right)>\left(1-\alpha_{i j}^{\prime}\right)\left(G_{j}-G_{j}^{\prime}\right)>0$,

yielding a contradiction. Hence, $G_{i}^{\prime} \leq G_{i}$. Notice that similarly, if $G_{j}>G_{j}^{\prime}$, then $G_{i}^{\prime}>G_{i}$ and hence, the same argument shows that $G_{j}^{\prime} \geq G_{j}$. Finally, the argument of Lemma 5 can be used to show that $U_{i}^{\prime} \geq U_{i}$ and $U_{j}^{\prime} \leq U_{j}$. 
Proposition 8 establishes that an increase in the flow of spillovers to jurisdiction $i$ reduces the public good contribution of that jurisdiction, and increases the public good provision of the other jurisdiction. This result is easy to interpret. An increase in $\alpha_{i j}$ results in a decrease in the entire best response function of jurisdiction $i$, while it leaves the best response function of jurisdiction $j$ unchanged. Hence, in equilibrium, the public good contribution of jurisdiction $i$ must go down. As best response functions are donward sloping, jurisdiction $j$ necessarily reacts by increasing its public good contribution. We note that this change always results in an increase in the utility of jurisdiction $i$ and a decrease in the utility of jurisdiction $j$. This last effect, while resting on a solid economic intuition, is somewhat puzzling, as one may expect that an increase in spillover flows always results in a better situation for all agents.

Unfortunately, the result of Proposition 8 cannot be extended beyond two jurisdictions. The following simple example shows that an increase in $\alpha_{i j}$ can actually result in an increase in the provision of public good of jurisdiction $i$ if one considers more than two jurisdictions.

Example 3 Let $n=3, u(c, \gamma)=c+\log \gamma, m_{1}=m, m_{2}=m_{3}=\frac{1-m}{2}, A=$ $\left[\begin{array}{lll}1 & \alpha & 1 \\ 1 & 1 & 0 \\ 1 & 0 & 1\end{array}\right]$ with $(1-m)(\alpha+1)>2 m>1-m$.

There exists an interior equilibrium where

$$
\begin{aligned}
& G_{1}=\frac{(1-m)(\alpha+1)-2 m}{2 \alpha}, \\
& G_{2}=G_{3}=\frac{2 m-(1-m)}{2 \alpha}
\end{aligned}
$$

One can easily check that an increase in $\alpha$ leads to a decrease in $G_{2}$ and $G_{3}$ and an increase in $G_{1}$.

Example 3 thus shows that the effect of an increase in the flow from jurisdiction 2 to jurisdiction 1 depends on the entire structure of spillovers. The presence of a third jurisdiction increases the complexity of the interaction, and it is in general impossible to sign the effect of changes in spillovers with general spillover matrices. 


\section{Conclusion}

The analysis of this paper highlights the complex interaction between jurisdiction sizes and the matrix of spillovers in the determination of equilibrium. This complexity was already noted by Williams (1966, p. 19) who wrote:

In a setting in which local governments are left free to make their own independent decisions about the supply of public goods, the complex interactions that occur even in highly simplified situations make it impossible to predict a priori whether undersupply or oversupply will generally result.

In spite of this complexity, our analysis gives rise to a few very simple observations. First, the model with symmetric spillovers isolates the role of jurisdiction sizes in the determination of equilibrium, and shows that larger jurisdictions, which provide more public goods, are more prone to increase their provision when the spillover parameter increases. The main message from the model with general spillovers is that multiple equilibria are likely to emerge, and that the effect of an increase in spillovers on the provision of public goods of one jurisdiction depends on the entire structure of spillovers.

We are aware of the fact that our model rests on several limitative assumptions. We suppose that public goods provided in different jurisdictions are perfect substitutes and focus attention on positive spillovers; we assume away interjurisdictional transfers and consider that the objective of every jurisdiction is to maximize the utility of its members. We also assume that preferences of agents are homogeneous and they have identical incomes. We believe that a richer model, where some of these assumptions are relaxed, deserves further study. In particular, altough it will complicate the analysis considerably, relaxing the preference and/or income homogenity assumption in a tractable manner will allow addressing questions regarding how spillovers across jurisdictions may affects stratification (sorting) of agents into different jurisdictions according to their preferences and/or income. 


\section{References}

Bergstrom, T. , L. Blume and H. Varian (1986), On the Private Provision of Public Goods, Journal of Public Economics, v. 29, iss. 1, pp. 25-49.

Bergstrom, T., L. Blume and H. Varian (1992), Uniqueness of Nash Equilibrium in Private Provision of Public Goods: An Improved Proof, Journal of Public Economics, v. 49, iss. 3, pp. 391-92.

Besley, T. and S. Coate (2003), Centralized versus Decentralized Provision of Local Public Goods: A Political Economy Approach, Journal of Public Economics, v. 87, iss. 12, pp. 2611-37.

Bjorvatn, K. and G. Schjelderup (2002), Tax Competition and International Public Goods, International Tax and Public Finance, v. 9, iss. 2, pp. 111-20.

Bloch, F. and U. Zenginobuz (2004), Tiebout Equilibria in Local Public Good Economies with Spillovers, forthcoming, Journal of Public Economics.

Boskin, M. (1973), Local Government Tax and Product Competition and the Optimal Provision of Public Goods, Journal of Political Economy, Vol. 81, No. 1, pp. 203-210.

Brainard, W. and F. Dolbaer (1967), The Possibility of Oversupply of Local "Public" Goods: A Critical Note, Journal of Political Economy, Vol. 75, No. 1, pp. 86-90.

Cremer, J. and T. Palfrey (2003), A Voting Model of Federal Standards with Externalities, California Institute of Technology, Division of the Humanities and Social Sciences, Working Papers: 1171.

Lockwood, B. (2002), Distributive Politics and the Costs of Centralization, Review of Economic Studies, v. 69, iss. 2, pp. 313-37.

Oates, W. (1972), Fiscal Federalism, New York: Harcourt Brace Jovanovich.

Redoano, M. and K. Scharf (2004), The Political Economy of Policy Centralization: Direct versus Representative Democracy, Journal of Public Economics, v. 88, iss. 3-4, pp. 799-817.

Vives, X. (2000), Oligopoly Pricing: Old Ideas and New Tools, Cambridge: MIT Press. 
Williams, A. (1966), The Optimal Provision of Public Goods in a System of Local Government, Journal of Political Economy, Vol. 74, No. 1, pp. 18-33. 